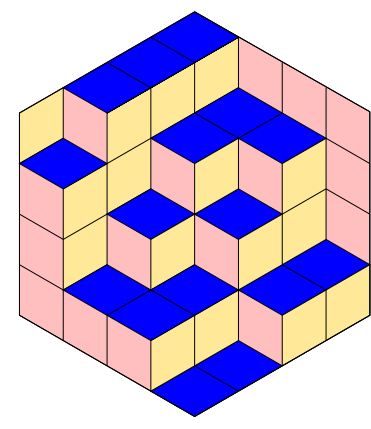

Zachary Hamaker, Oliver Pechenik, David E Speyer \& Anna Weigandt Derivatives of Schubert polynomials and proof of a determinant conjecture of Stanley Volume 3, issue 2 (2020), p. 301-307.

<http://alco.centre-mersenne.org/item/ALCO_2020__3_2_301_0>

(c) The journal and the authors, 2020. Some rights reserved.

(c) BY This article is licensed under the

Creative Commons ATtribution 4.0 InTERnational License.

http://creativecommons.org/licenses/by/4.0/

Access to articles published by the journal Algebraic Combinatorics on the website http://alco.centre-mersenne.org/ implies agreement with the Terms of Use (http://alco.centre-mersenne.org/legal/).

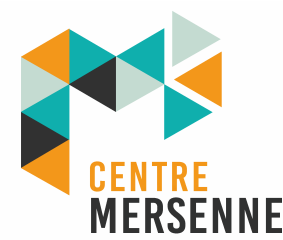

Algebraic Combinatorics is member of the Centre Mersenne for Open Scientific Publishing www.centre-mersenne.org 


\title{
Derivatives of Schubert polynomials and proof of a determinant conjecture of Stanley
}

\author{
Zachary Hamaker, Oliver Pechenik, David E Speyer \& Anna \\ Weigandt
}

\begin{abstract}
We study the action of a differential operator on Schubert polynomials. Using this action, we first give a short new proof of an identity of I. Macdonald (1991). We then prove a determinant conjecture of R. Stanley (2017). This conjecture implies the (strong) Sperner property for the weak order on the symmetric group, a property recently established by C. Gaetz and Y. Gao (2019).
\end{abstract}

\section{INTRODUCTION}

This paper is motivated by a conjecture of R. Stanley [10, Conjecture 2.2]. Let $\mathcal{S}_{n}$ be the symmetric group ${ }^{(1)}$ with its standard generating set $S=\left\{s_{1}, s_{2}, \ldots, s_{n-1}\right\}$, and let $\mathcal{S}_{n}(\ell)$ denote the subset of those permutations of (Coxeter) length $\ell$. For $1 \leqslant \ell \leqslant\left(\begin{array}{c}n \\ 2\end{array}\right)$, let $M_{\ell}$ be the matrix with rows indexed by $\mathcal{S}_{n}(\ell-1)$ and columns indexed by $\mathcal{S}_{n}(\ell)$, where the entry in position $(u, v)$ is

$$
M_{\ell}[u, v]= \begin{cases}k, & \text { if } v=u s_{k} \text { and } \\ 0, & \text { if } u^{-1} v \notin S .\end{cases}
$$

For $\ell \leqslant\left(\begin{array}{l}n \\ 2\end{array}\right)-\ell$, the product $\widetilde{M}^{(\ell)}=M_{\ell+1} M_{\ell+2} \cdots M_{\left(\begin{array}{c}n \\ 2\end{array}\right)-\ell}$ is a square matrix with rows indexed by $\mathcal{S}_{n}(\ell)$ and columns indexed by $\left.\mathcal{S}_{n}\left(\begin{array}{c}n \\ 2\end{array}\right)-\ell\right)$. Stanley conjectures an explicit formula for $\operatorname{det} \widetilde{M}^{(\ell)}$, which implies that $\widetilde{M}^{(\ell)}$ is invertible. A motivation for this conjecture is that $M_{\ell(u)+1} M_{\ell(u)+2} \cdots M_{\ell(v)}[u, v]$ is nonzero if and only if $u \leqslant v$ in weak order. Hence, by standard linear-algebraic arguments (cf. $[9,10])$, showing that $\operatorname{det} \widetilde{M}^{(\ell)}$ is nonzero implies that the weak order on the symmetric group has the (strong) Sperner property.

Recently, C. Gaetz and Y. Gao [5] proved the invertibility of Stanley's matrix by constructing an action of the Lie algebra $\mathfrak{s l}_{2}$. We give a new proof of invertibility by proving Stanley's determinant conjecture. Our proof also involves an $\mathfrak{s l}_{2}$ representation, but relies on a new identity for derivatives of Schubert polynomials.

Manuscript received 4th January 2019, revised 6th August 2019, accepted 9th August 2019. KEYwORDS. Sperner property, weak order, Schubert polynomial, Macdonald identity.

ACKNowledgements. OP was partially supported by a Mathematical Sciences Postdoctoral Research Fellowship (\#1703696) from the National Science Foundation. DES was partially supported by NSF Grant DMS-1600223.

${ }^{(1)}$ Interpreting $\mathcal{S}_{n}$ as the group of bijections from $\{1,2, \ldots, n\}$ to itself, we define multiplication by $w v:=v \circ w$. 
The generator $s_{i}$ acts on Poly $_{n}=\mathbb{C}\left[x_{1}, \ldots, x_{n}\right]$ by

$$
s_{i} \cdot f\left(x_{1}, \ldots, x_{i}, x_{i+1}, \ldots, x_{n}\right)=f\left(x_{1}, \ldots, x_{i+1}, x_{i}, \ldots, x_{n}\right) .
$$

We define the Newton divided difference operators on Poly ${ }_{n}$ by

$$
N_{i}(f)=\frac{f-s_{i} \cdot f}{x_{i}-x_{i+1}} .
$$

(We avoid the more standard notation $\partial_{i}$ because of potential confusion with partial derivatives.) For $w \in \mathcal{S}_{n}$, the Schubert polynomials $\mathfrak{S}_{w}$ are defined by the recurrence

$$
\mathfrak{S}_{s_{k} w}=N_{k} \mathfrak{S}_{w} \text { for } \ell\left(s_{k} w\right)<\ell(w)
$$

with $\mathfrak{S}_{w_{0}}=x_{1}^{n-1} x_{2}^{n-2} \cdots x_{n-1}$. For background on Schubert polynomials, we refer the reader to $[6,7]$.

We consider the differential operator $\nabla=\sum_{i=1}^{n} \frac{\partial}{\partial x_{i}}$. Our key result is the following:

Proposition 1.1. For $w \in \mathcal{S}_{n}$, we have

$$
\nabla\left(\mathfrak{S}_{w}\right)=\sum_{\ell\left(w s_{k}\right)<\ell(w)} k \mathfrak{S}_{w s_{k}} .
$$

Thus, Stanley's $M$-matrices are the matrices of the operator $\nabla$ in the basis of Schubert polynomials.

We first apply Proposition 1.1 to give a short new proof of a theorem of Macdonald [6, (6.11)]. We then use Proposition 1.1 to prove Stanley's conjecture.

Theorem 1.2 (Conjectured by Stanley [10, Conjecture 2.2]). For $\ell \leqslant\left(\begin{array}{l}n \\ 2\end{array}\right)-\ell$,

$$
\operatorname{det} \widetilde{M}^{(\ell)}= \pm \prod_{k=0}^{\ell}\left((\ell-k+1)(\ell-k+2) \cdots\left(\left(\begin{array}{l}
n \\
2
\end{array}\right)-\ell-k\right)\right)^{\left|\mathcal{S}_{n}(k)\right|-\left|\mathcal{S}_{n}(k-1)\right|}
$$

We write \pm because we have not specified an order on the rows and columns of each $M_{i}$. In more recent work, C. Gaetz and Y. Gao [4] build on the results here to obtain, among other consequences, an explicit description of the Smith normal form of $\widetilde{M}^{(\ell)}$.

\section{Proof of Proposition 1.1 And A Macdonald identity}

We start with a straightforward lemma.

LEMma 2.1. The differential operator $\nabla$ commutes with $N_{i}$ for all $i$. That is, for any $f \in$ Poly $_{n}$, we have

$$
\nabla\left(N_{i}(f)\right)=N_{i}(\nabla(f))
$$

Proof. If $j \notin\{i, i+1\}$, then

$$
\frac{\partial}{\partial x_{j}} N_{i}(f)=\frac{1}{x_{i}-x_{i+1}}\left(\frac{\partial}{\partial x_{j}}(f)-\frac{\partial}{\partial x_{j}}\left(s_{i} \cdot f\right)\right) .
$$

If $j=i$, then

$$
\frac{\partial}{\partial x_{i}} N_{i}(f)=\frac{1}{x_{i}-x_{i+1}}\left(\frac{\partial}{\partial x_{i}}(f)-\frac{\partial}{\partial x_{i}}\left(s_{i} \cdot f\right)\right)-\frac{f-s_{i} \cdot f}{\left(x_{i}-x_{i+1}\right)^{2}} .
$$

Similarly, if $j=i+1$, then

$$
\frac{\partial}{\partial x_{i+1}} N_{i}(f)=\frac{1}{x_{i}-x_{i+1}}\left(\frac{\partial}{\partial x_{i+1}}(f)-\frac{\partial}{\partial x_{i+1}}\left(s_{i} \cdot f\right)\right)+\frac{f-s_{i} \cdot f}{\left(x_{i}-x_{i+1}\right)^{2}} .
$$


Therefore,

$$
\begin{aligned}
\nabla\left(N_{i}(f)\right) & =\frac{f-s_{i} \cdot f}{\left(x_{i}-x_{i+1}\right)^{2}}-\frac{f-s_{i} \cdot f}{\left(x_{i}-x_{i+1}\right)^{2}}+\sum_{j=1}^{n} \frac{1}{x_{i}-x_{i+1}}\left(\frac{\partial}{\partial x_{j}}(f)-\frac{\partial}{\partial x_{j}}\left(s_{i} \cdot f\right)\right) \\
& =\frac{\sum_{j=1}^{n} \frac{\partial}{\partial x_{j}}(f)-\sum_{j=1}^{n} \frac{\partial}{\partial x_{j}}\left(s_{i} \cdot f\right)}{x_{i}-x_{i+1}}=\frac{\nabla(f)-\nabla\left(s_{i} \cdot f\right)}{x_{i}-x_{i+1}} .
\end{aligned}
$$

Since $\nabla$ is $\mathcal{S}_{n}$-invariant, it commutes with $s_{i}$, so we obtain

$$
\frac{\nabla(f)-\nabla\left(s_{i} \cdot f\right)}{x_{i}-x_{i+1}}=\frac{\nabla(f)-s_{i} \cdot \nabla(f)}{x_{i}-x_{i+1}}=N_{i}(\nabla(f)) .
$$

Thus, $\nabla\left(N_{i}(f)\right)=N_{i}(\nabla(f))$, as desired.

Proof of Proposition 1.1. We first verify Proposition 1.1 in the case that $w=w_{0}$. Since $\mathfrak{S}_{w_{0}}=x_{1}^{n-1} x_{2}^{n-2} \cdots x_{n-1}$, we have

$$
\begin{aligned}
\nabla\left(\mathfrak{S}_{w_{0}}\right) & =\sum_{j=1}^{n-1}(n-j) x_{1}^{n-1} x_{2}^{n-2} \cdots x_{j-1}^{n-j+1} x_{j}^{n-j-1} x_{j+1}^{n-j-1} \cdots x_{n-1} \\
& =\sum_{k=1}^{n-1} k x_{1}^{n-1} x_{2}^{n-2} \cdots x_{n-k-1}^{k+1} x_{n-k}^{k-1} x_{n-k+1}^{k-1} \cdots x_{n-1} .
\end{aligned}
$$

But also

$$
\mathfrak{S}_{w_{0} s_{k}}=\mathfrak{S}_{s_{n-k} w_{0}}=N_{n-k}\left(\mathfrak{S}_{w_{0}}\right)=x_{1}^{n-1} x_{2}^{n-2} \cdots x_{n-k-1}^{k+1} x_{n-k}^{k-1} x_{n-k+1}^{k-1} \cdots x_{n-1} .
$$

Comparing these equations gives

$$
\nabla\left(\mathfrak{S}_{w_{0}}\right)=\sum_{k=1}^{n-1} k \mathfrak{S}_{w_{0} s_{k}}
$$

Consider an arbitrary permutation $w$. Let $r=\left(\begin{array}{c}n \\ 2\end{array}\right)-\ell(w)$ and write $w=$ $s_{i_{1}} s_{i_{2}} \cdots s_{i_{r}} w_{0}$. By Lemma 2.1, we have

$(\star) \quad \nabla\left(\mathfrak{S}_{w}\right)=\nabla N_{i_{1}} \cdots N_{i_{r}}\left(\mathfrak{S}_{w_{0}}\right)=N_{i_{1}} \cdots N_{i_{r}} \nabla\left(\mathfrak{S}_{w_{0}}\right)=N_{i_{1}} \cdots N_{i_{r}} \sum_{k=1}^{n-1} k \mathfrak{S}_{w_{0} s_{k}}$.

Hence,

$$
N_{i_{1}} N_{i_{2}} \cdots N_{i_{r}}\left(\mathfrak{S}_{w_{0} s_{k}}\right)= \begin{cases}\mathfrak{S}_{s_{i_{1}} \cdots s_{i_{r}} w_{0} s_{k}}, & \text { if } \ell\left(w s_{k}\right)=\left(\begin{array}{l}
n \\
2
\end{array}\right)-r-1=\ell(w)-1 \\
0, & \text { otherwise. }\end{cases}
$$

Since $\mathfrak{S}_{s_{i_{1}} \cdots s_{i_{r}} w_{0} s_{k}}=\mathfrak{S}_{w s_{k}}$, Equation $(\star)$ then becomes

$$
\nabla\left(\mathfrak{S}_{w}\right)=\sum_{\ell\left(w s_{k}\right)=\ell(w)-1} k \mathfrak{S}_{w s_{k}},
$$

as desired.

Proposition 1.1 yields a short proof of an identity of Macdonald [6, (6.11)]. Another proof of this result was given by S. Fomin and R. Stanley in terms of nilCoxeter algebras [3], while a bijective proof was given by S. Billey, A. Holroyd and B. Young [2]. A reduced word for $w \in \mathcal{S}_{n}$ is a tuple $a=\left(a_{1}, \ldots, a_{\ell(w)}\right)$ such that $w=s_{a_{1}} s_{a_{2}} \cdots s_{a_{\ell(w)}}$. We write $R(w)$ for the set of reduced words of $w$. 
Theorem 2.2 (Macdonald $[6,(6.11)]$ ). Let $w \in \mathcal{S}_{n}$ with $\ell(w)=k$. Then

$$
\frac{1}{k !} \sum_{a \in R(w)} a_{1} a_{2} \cdots a_{k}=\mathfrak{S}_{w}(1,1, \ldots, 1) .
$$

Proof. For any monomial $\mu$ of degree $k$, we have $\nabla^{k}(\mu)=k$ !. Since $\mathfrak{S}_{w}$ is homogeneous of degree $k$, we then see $\nabla^{k}\left(\mathfrak{S}_{w}\right)=k ! \mathfrak{S}_{w}(1,1, \ldots, 1)$. On the other hand, by Proposition 1.1,

$$
\nabla^{k}\left(\mathfrak{S}_{w}\right)=\sum_{a \in R(w)} a_{1} a_{2} \cdots a_{k}
$$

\section{A VECTOR SPACE OF POLYNOMials}

Let $W \subset$ Poly $_{n}$ be the span of the monomials of the form $x_{1}^{a_{1}} x_{2}^{a_{2}} \cdots x_{n}^{a_{n}}$ with $0 \leqslant$ $a_{j} \leqslant n-j$. (In particular, the exponent of $x_{n}$ is required to be 0 , so the variable $x_{n}$ does not occur in any polynomial in $W$.) Let $W_{\ell}$ be the subspace of $W$ spanned by monomials of degree $\ell$. We will need the following lemma.

Lemma 3.1 ([1, Proof of Corollary 3.9]). For $w \in \mathcal{S}_{n}$, the Schubert polynomial $\mathfrak{S}_{w}$ lies in $W$. If we choose a term order with $x_{n}>x_{n-1}>\cdots>x_{1}$, then the leading term of $\mathfrak{S}_{w}$ is

$$
\prod_{j=1}^{n} x_{j}^{\#\{k: k>j, w(k)<w(j)\}} .
$$

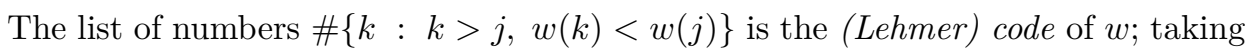
the code is a bijection between $\mathcal{S}_{n}$ and $\left\{\left(a_{1}, \ldots, a_{n}\right) \in \mathbb{Z}^{n}: 0 \leqslant a_{j} \leqslant n-j\right\}$ (see, e.g. [7, Proposition 2.1.2]). Thus, Lemma 3.1 implies that the Schubert polynomials have distinct leading terms and we deduce:

COROLlary 3.2. The Schubert polynomials $\mathfrak{S}_{w}$ for $w \in \mathcal{S}_{n}$ are a basis for $W$. The change of basis matrix between $\left\{\mathfrak{S}_{w}: \ell(w)=k\right\}$ and $\left\{x_{1}^{a_{1}} x_{2}^{a_{2}} \cdots x_{n}^{a_{n}}: 0 \leqslant a_{j} \leqslant\right.$ $\left.n-j, \sum a_{j}=k\right\}$ has determinant \pm 1 .

Proposition 1.1 shows that $\nabla: W_{\ell} \rightarrow W_{\ell-1}$, in the Schubert basis, is represented by the matrix $M_{\ell}$. Therefore, to prove Theorem 1.2 , we must compute

$$
\operatorname{det}\left(W_{\left(\begin{array}{c}
n \\
2
\end{array}\right)-\ell} \stackrel{\nabla^{\left(\begin{array}{c}
n \\
2
\end{array}\right)-2 \ell}}{\longrightarrow} W_{\ell}\right)
$$

in the Schubert basis. By Corollary 3.2, we may compute this determinant instead in the monomial basis. For the remainder of this note, Schubert polynomials disappear and our goal is to compute the determinant of $\nabla^{j}$ acting with respect to the monomial basis.

We would prefer to have a map from a vector space to itself, so that we could speak of its determinant without any reference to bases. There is a simple bijection between the monomial bases of $W_{k}$ and $W_{\left(\begin{array}{c}n \\ 2\end{array}\right)-k}$, taking $\prod x_{j}^{a_{j}}$ to $\prod x_{j}^{n-j-a_{j}}$. For reasons that will become clear in Section 4 , we prefer to twist this map by $(-1)^{k}$, so we define $J$ to be the linear endomorphism of $W$ with

$$
J\left(\prod x_{j}^{a_{j}}\right)=(-1)^{\sum a_{j}} \prod x_{j}^{n-j-a_{j}} .
$$


Note that $J$ has determinant \pm 1 in the monomial basis and that $\nabla^{\left(\begin{array}{l}n \\ 2\end{array}\right)-2 \ell} \circ J$ maps $W_{\ell}$ to itself. To finish our proof of Theorem 1.2, it remains to establish

$$
\operatorname{det} \tau= \pm \prod_{k=0}^{\ell}\left((\ell-k+1)(\ell-k+2) \cdots\left(\left(\begin{array}{c}
n \\
2
\end{array}\right)-\ell-k\right)\right)^{\left|\mathcal{S}_{n}(k)\right|-\left|\mathcal{S}_{n}(k-1)\right|}
$$

where $\tau$ is the linear transformation $W_{\ell} \stackrel{\nabla^{\left(\begin{array}{c}n \\ 2\end{array}\right)-2 \ell}{ }_{\circ J}}{\longrightarrow} W_{\ell}$. We now turn to this task.

\section{4. $\mathrm{SL}_{2}$-Representations AND A PROOF OF TheOREM 1.2}

In this section, we discuss some representations of the Lie group $\mathrm{SL}_{2}$ and its Lie algebra $\mathfrak{s l}_{2}$. Our approach to proving Theorem 1.2 via these representations mirrors the general framework of R. Proctor from [8]; however, we provide a self-contained argument. We will denote group actions by variants of the letter $\rho$ and the corresponding Lie algebra actions by variants of $\sigma$. We write the standard basis of $\mathfrak{s l}_{2}$ as

$$
F=\left[\begin{array}{ll}
0 & 0 \\
1 & 0
\end{array}\right], \quad H=\left[\begin{array}{cc}
1 & 0 \\
0 & -1
\end{array}\right], \quad E=\left[\begin{array}{ll}
0 & 1 \\
0 & 0
\end{array}\right]
$$

and define the element

$$
J=\left[\begin{array}{cc}
0 & 1 \\
-1 & 0
\end{array}\right] \in \mathrm{SL}_{2}
$$

Let $V_{k}$ be the $(k+1)$-dimensional irreducible representation of $\mathrm{SL}_{2}$ and $\mathfrak{s l}_{2}$; we write $\rho_{k}$ and $\sigma_{k}$ for the action maps $\rho_{k}: \mathrm{SL}_{2} \rightarrow \mathrm{GL}\left(V_{k}\right)$ and $\sigma_{k}: \mathfrak{s l}_{2} \rightarrow \operatorname{End}\left(V_{k}\right)$.

One usually describes $V_{k}$ as the natural action on degree $k$ polynomials in two variables. For our purposes, it is more convenient to describe $V_{k}$ as an action on polynomials of degree $\leqslant k$ in one variable $x$. We have

$$
\begin{array}{rr}
\sigma_{k}(F)\left(x^{j}\right) & =j x^{j-1}, \\
\sigma_{k}(H)\left(x^{j}\right) & =(2 j-k) x^{j}, \\
\sigma_{k}(E)\left(x^{j}\right) & =(k-j) x^{j+1}, \\
\rho_{k}(J)\left(x^{j}\right) & =(-1)^{j} x^{k-j} .
\end{array}
$$

In particular, for any polynomial $f$, we have $\sigma_{k}(F)(f)=\frac{\mathrm{d} f}{\mathrm{~d} x}$.

Identify the vector space $W$ from Section 3 with $V_{n-1} \otimes V_{n-2} \otimes \cdots \otimes V_{0}$ by identifying $x_{1}^{a_{1}} x_{2}^{a_{2}} \cdots x_{n}^{a_{n}}$ with $x^{a_{1}} \otimes x^{a_{2}} \otimes \cdots \otimes x^{a_{n}}$. We let $\mathrm{SL}_{2}$ and $\mathfrak{s l}_{2}$ act on this tensor product in the standard way, and denote these actions by $\sigma_{W}$ and $\rho_{W}$. We note that $W_{\ell}$ is the $2 \ell-\left(\begin{array}{l}n \\ 2\end{array}\right)$ weight space, i.e. the $2 \ell-\left(\begin{array}{l}n \\ 2\end{array}\right)$ eigenspace of $\sigma_{W}(H)$.

We have

$$
\sigma_{W}(F)=\sum_{k=1}^{n} \operatorname{Id} \otimes \operatorname{Id} \otimes \cdots \otimes \sigma_{n-k}(F) \otimes \cdots \otimes \operatorname{Id}
$$

where $\sigma_{n-k}(F)$ occurs in the $k$-th position. Therefore,

$$
\sigma_{W}(F) \cdot f=\sum_{k=1}^{n} \frac{\partial}{\partial x_{k}} f=\nabla f .
$$

Similarly,

$$
\rho_{W}(J)=\rho_{n-1}(J) \otimes \rho_{n-2}(J) \otimes \cdots \otimes \rho_{0}(J),
$$

so

$$
\rho_{W}(J) \cdot\left(\prod_{j=1}^{n} x_{j}^{a_{j}}\right)=\prod_{j=1}^{n}(-1)^{a_{j}} x_{j}^{n-j-a_{j}}=(-1)^{\sum a_{j}} \prod_{j=1}^{n} x_{j}^{n-j-a_{j}}=J\left(\prod_{j=1}^{n} x_{j}^{a_{j}}\right) .
$$


Thus, our goal of computing $\operatorname{det}\left(\nabla^{\left(\begin{array}{c}n \\ 2\end{array}\right)-2 \ell} \circ J\right)$ on $W_{\ell}$ is the same as computing the determinant of $\sigma_{W}(F)\left(\begin{array}{c}n \\ 2\end{array}\right)-2 \ell \rho_{W}(J)$ as a map from the $2 \ell-\left(\begin{array}{c}n \\ 2\end{array}\right)$ weight space of $V_{n-1} \otimes V_{n-2} \otimes \cdots \otimes V_{0}$ to itself.

This is a standard computation. By comparing dimensions of weight spaces,

$$
V_{n-1} \otimes V_{n-2} \otimes \cdots \otimes V_{0} \cong \bigoplus_{0 \leqslant k \leqslant\left(\begin{array}{c}
n \\
2
\end{array}\right)-k} V_{\left(\begin{array}{c}
n \\
2
\end{array}\right)-2 k}^{\oplus\left|\mathcal{S}_{n}(k)\right|-\left|\mathcal{S}_{n}(k-1)\right|}
$$

Thus, Theorem 1.2 comes down to showing that $\sigma_{W}(F)^{\left(\begin{array}{c}n \\ 2\end{array}\right)-2 \ell} \rho_{W}(J)$ as a map from the $2 \ell-\left(\begin{array}{l}n \\ 2\end{array}\right)$ weight space of $V_{\left(\begin{array}{c}n \\ 2\end{array}\right)-2 k}$ to itself is $\pm(\ell-k+1)(\ell-k+2) \cdots\left(\left(\begin{array}{l}n \\ 2\end{array}\right)-\ell-k\right)$. Consulting the formulas from Equation $(\dagger)$, one sees that

$$
\rho_{\left(\begin{array}{c}
n \\
2
\end{array}\right)-2 k}(J) x^{\ell-k}=(-1)^{\ell-k} x^{\left(\begin{array}{c}
n \\
2
\end{array}\right)-2 k-(\ell-k)}=(-1)^{\ell-k} x^{\left(\begin{array}{c}
n \\
2
\end{array}\right)-\ell-k}
$$

and

$$
\begin{aligned}
\sigma_{\left(\begin{array}{c}
n \\
2
\end{array}\right)-2 k}(F)^{\left(\begin{array}{c}
n \\
2
\end{array}\right)-2 \ell} x^{\left(\begin{array}{c}
n \\
2
\end{array}\right)-\ell-k} & =\left(\frac{\mathrm{d}}{\mathrm{d} x}\right)^{\left(\begin{array}{c}
n \\
2
\end{array}\right)-2 \ell} x^{\left(\begin{array}{c}
n \\
2
\end{array}\right)-\ell-k} \\
& =(\ell-k+1)(\ell-k+2) \cdots\left(\left(\begin{array}{l}
n \\
2
\end{array}\right)-\ell-k\right) x^{\ell-k} .
\end{aligned}
$$

Theorem 1.2 follows.

Acknowledgements. We are grateful for helpful conversations with Ben Elias, Sergey Fomin and Benjamin Young. We would also like to thank two anonymous referees for helpful comments.

\section{REFERENCES}

[1] Nantel Bergeron and Sara C. Billey, RC-graphs and Schubert polynomials, Experiment. Math. 2 (1993), no. 4, 257-269.

[2] Sara C. Billey, Alexander E. Holroyd, and Benjamin J. Young, A bijective proof of Macdonald's reduced word formula, Algebraic Combin. 2 (2019), no. 2, 217-248.

[3] Sergey Fomin and Richard P. Stanley, Schubert polynomials and the nil-Coxeter algebra, Adv. Math. 103 (1994), no. 2, 196-207.

[4] Christian Gaetz and Yibo Gao, A combinatorial duality between the weak and strong Bruhat orders, https://arxiv.org/abs/1812.05126, 2018.

[5] _ A combinatorial $\mathfrak{s l}_{2}$-action and the Sperner property for the weak order, Proc. Amer. Math. Soc., to appear, https://arxiv.org/abs/1811.05501, 2019.

[6] Ian Grant Macdonald, Notes on Schubert polynomials, vol. 6, Publications du LACIM, Université du Québec à Montréal, 1991.

[7] Laurent Manivel, Symmetric functions, Schubert polynomials and degeneracy loci, SMF/AMS Texts and Monographs, vol. 6, American Mathematical Society, Providence, RI; Société Mathématique de France, Paris, 2001, Translated from the 1998 French original by John R. Swallow, Cours Spécialisés [Specialized Courses], 3.

[8] Robert A. Proctor, Product evaluations of Lefschetz determinants for Grassmannians and of determinants of multinomial coefficients, J. Comb. Theory, Ser. A 54 (1990), no. 2, 235-247.

[9] Richard P. Stanley, Weyl groups, the hard Lefschetz theorem, and the Sperner property, SIAM J. Algebraic Discrete Methods 1 (1980), no. 2, 168-184.

[10] _ Some Schubert shenanigans, https://arxiv.org/abs/1704.00851, 2017. 


\section{Derivatives of Schubert polynomials}

Zachary Hamaker, Department of Mathematics, University of Florida, Gainesville, FL 32601, USA E-mail : zhamaker@ufl.edu

Oliver Pechenik, Department of Mathematics, University of Michigan, Ann Arbor, MI 48109, USA

E-mail : pechenik@umich.edu

DAvid E Speyer, Department of Mathematics, University of Michigan, Ann Arbor, MI 48109, USA E-mail : speyer@umich.edu

Anna Weigandt, Department of Mathematics, University of Michigan, Ann Arbor, MI 48109, USA E-mail : weigandt@umich.edu 